\title{
THE TOPOLOGICAL COMPLEMENTATION PROBLEM
}

\author{
BY A. K. STEINER ${ }^{1,2}$
}

\author{
Communicated by R. S. Pierce, August 30, 1965
}

Let $\Sigma$ be the lattice of all topologies definable on an arbitrary set $E$. Then $\Sigma$ is a complete lattice with the trivial topology, $\{\varnothing, E\}$, as the least element and the discrete topology, $P(E)$, as the greatest element.

The problem of complementation in the lattice $\Sigma$ has been outstanding for some time although several investigators have provided partial solutions. Hartmanis [6] first showed that $\Sigma$ was a complemented lattice if the set $E$ was finite and Gaifman [4] proved $\Sigma$ was complemented if $E$ was countable. Berri [1], using the results of Gaifman, was able to provide complements for certain special topologies such as a topological group with a dense, nonopen, countable subgroup.

It is the purpose of this paper to introduce the lattice of principal topologies, and to establish that the lattice $\Sigma$ of all topologies on a set $E$ is complemented.

The following theorems are stated without proof. The full details will be published elsewhere.

1. Principal topologies. A topology $\tau \in \Sigma$ is called an ultraspace if the only topology finer than $\tau$ is the discrete topology. Fröhlich [3] shows that every topology $\tau$ is the infimum of ultraspaces finer than $\tau$. For a filter $\mathfrak{F}$ on $E$ and a point $x \in E$, Fröhlich defined $\mathfrak{S}(x, \mathfrak{F})$ to be the family of sets $P(E-\{x\}) \cup \mathfrak{F}$, which is a topology. He proved the ultraspaces are the topologies of the form $\mathfrak{S}(x, \mathfrak{u})$ where $x \in E$ and $\mathfrak{U}$ is an ultrafilter on $E$ different from the principal ultrafilter at $x, \mathfrak{u}(x)$. The set of ultraspaces may be divided into two classes each of which generates a sublattice of $\Sigma$. One of these sublattices consists of all $T_{1}$-topologies. The other is called the lattice of principal topologies.

Every topology $\tau$ on $E$ is the infimum of all ultraspaces on $E$ finer than $\tau$. If also $\tau=\inf \{\subseteq(x, \mathfrak{U}(y)) \mid \subseteq(x, \mathfrak{U}(y)) \geqq \tau\}$ then $\tau$ will be called a principal topology.

\footnotetext{
1 These results are part of the author's doctoral dissertation, submitted to the University of New Mexico.

2 This research was partially supported by the National Science Foundation, Grant GP-2214.
} 
THEOREM 1.1. The principal topologies form a sublattice of the lattice $\Sigma$.

The lattice of principal topologies on $E$ will be denoted $\Pi$. The lattice $\Pi$ is a complete lattice but is not a complete sublattice of $\Sigma$.

THEOREM 1.2. A topology $\tau$ on $E$ is a principal topology if and only if for each $x \in E$ there is a minimal element in $\tau$ containing $x$.

A principal topology then, is one which is closed under arbitrary intersections.

The family $g$ of pre-order relations forms a complete lattice with $E \times E$ as the greatest element and $\Delta=\{(x, x) \mid x \in E\}$ as the least element.

A relation $G$ defines a topology $\tau_{\theta}$ on $E$ : A set $S \subset E$ is open if and only if for each $x \in S$, if $(x, y) \in G$ then $y \in S$. The topology $\tau_{G}$ determined by the relation $G$ is a principal topology.

THEOREM 1.3. There is a one-to-one correspondence between principal topologies in $\Pi$ and pre-order relations in $\mathrm{g}$.

THEOREM 1.4. The lattice $\Pi$ of principal topologies is anti-isomorphic

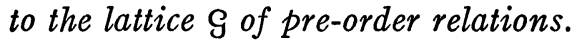

THEOREM 1.5. The lattice $G$ of pre-order relations on a set $E$ is a complemented lattice.

It follows from Theorem 1.4 that $\Pi$ is a complemented lattice. Since every topology on a finite set is a principal topology, this result extends the result of Hartmanis [6].

2. Complementation of the lattice $\Sigma$. A topology $\tau \in \Sigma$ has a principal complement if it has a lattice complement which is a principal topology.

Gaifman [5] proved that if every $T_{1}$-topology on a set has a complement then every topology on that set has a complement. Modifying his proof we obtain

THEOREM 2.1. If every $T_{1}$-topology on a set $E$ has a principal complement, then every topology on $E$ has a principal complement.

THEOREM 2.2. Let $\tau$ be a topology on a set $E=E_{1} \cup E_{2}$ where $E_{1} \cap E_{2}$ $=\varnothing$, such that $\tau \mid E_{1}$ and $\tau \mid E_{2}$ have principal lattice complements. Then $\tau$ has a principal complement.

THEOREM 2.3. If every topology ( $T_{1}$-topology) with no isolated points has a principal complement, then every topology ( $T_{1}$-topology) has a principal complement. 
The next theorem is an extension of a result of Berri [2]: A topology on a set $E$ has a complement if there is a decomposition of $E$ into countable sets such that no union of any proper subcollection is open.

TheOREM 2.4. Let $\tau$ be a topology on a set $E$ such that

(i) $E=\cup_{\alpha \in \theta} E_{\alpha}$, where $E_{\alpha}$ 's are pairwise disjoint,

(ii) $\tau \mid E_{\alpha}$ has a principal complement $\tau_{\alpha}^{\prime}$, for all $\alpha \in \theta$,

(iii) if $V \in \tau$ and if $V \neq E, \varnothing$, then $V$ is not the union of $E_{\alpha}$ 's.

Then $\tau$ has a principal complement $\tau^{\prime}$. If some $\tau_{\alpha}^{\prime}$ has an isolated point, so does $\tau^{\prime}$.

THEOREM 2.5. Let $\tau$ be a $T_{1}$-topology on a set $E$ containing a proper open set $S$ with at least two points, such that $\tau \mid S$ has a principal complement with an isolated point. Then $\tau$ has a principal complement with an isolated point.

THEOREM 2.6. $A$ T $T_{1}$-topology with no isolated points has a principal complement with an isolated point.

Now from Theorems 2.6, 2.3 and 2.1 we have

ThEOREM 2.7. The lattice of topologies on any set is complemented. Moreover, each topology has a principal complement.

\section{REFERENCES}

1. M. P. Berri, Lattice complements of certain topologies, Abstract 65 T-94, Notices Amer. Math. Soc. 12 (1965), 227.

2. - The complement of a topology for some topological groups, Fund. Math. (to appear).

3. O. Fröhlich, Das Halbordnungssystem der Topologischen Rdume auf einer Menge, Math. Ann. 156 (1964), 79-95.

4. H. Gaifman, The lattice of all topologies on a denumerable set, Abstract 61 T-161, Notices Amer. Math. Soc. 8 (1961), 356.

5. - Remarks on complementation in the lattice of all topologies (unpublished paper).

6. J. Hartmanis, On the lattice of topologies, Canad. J. Math. 10 (1958), 547-553.

UnIVERSITY OF NEW MeXico 